\title{
The Identification of Readiness in Implementating Business Intelligence Projects by Combining Interpretive Structural Modeling with Graph Theory and Matrix Approach
}

\author{
Vahid Farrokhi $^{1}$, László Pokorádi ${ }^{2}$, Sahar Bouini $^{3}$ \\ ${ }^{1}$ University of Debrecen, Doctoral School of Informatics, Kassai út 26, 4028 \\ Debrecen, Hungary, vahid.farrokhi@inf.unideb.hu \\ ${ }^{2}$ Óbuda University, Institute of Mechatronics and Vehicle Engineering, \\ Népszínház u. 8, 1081 Budapest, Hungary, pokoradi.laszlo@bgk.uni-obuda.hu \\ ${ }^{3}$ Institute for Advanced Studies in Basic Sciences,Department of Computer \\ Science and Information Technology, Prof. Yousef Sobouti Blvd., Zanjan, Iran, \\ s.bouini@iasbs.ac.ir
}

\begin{abstract}
In the past, decisions were made by senior management in organizations and they were based solely on personal experience, leading to increased risk in decision making. Nowadays, however, most companies are moving towards Business Intelligence (BI) systems. It is estimated that technology budgets dedicated to Business Intelligence in 2006, increased from $\$ 14$ to $\$ 20$ billion. Experiments have shown that the probability of failure in BI projects is high and evaluation before the start of implementation is important because if the company is not assessed, the implementation of BI projects can cause a waste of time and resources and the company will not achieve expected profits. The aim of this paper is to provide a method to evaluate the key factors for the successful implementation of BI projects and to determine the organization's index of assessing readiness before the implementation of BI projects. A series of technical and organizational key factors for the successful implementation of BI have been proposed in various literatures. These key factors will be evaluational ones for an organization. We apply a combination of Interpretive Structural Modeling (ISM) and Graph Theory and Matrix Approach (GTMA) on the factors to earn an indicator for evaluating the organization's readiness for implementing a BI project. We applied this method in two organizations for comparing and determined the organizations' readiness before the implementation of BI and found it to be very effective.
\end{abstract}

Keywords: Business Intelligence; Readiness Evaluation; Critical Success Factors; Interpretive Structural Modeling; Graph Theory and Matrix Approach 


\section{Introduction}

Information and knowledge have become the main wealth of organizations in the third millennium [1]. Commercial institutes and production units trying to utilize as much of their wealth in their critical decisions to gain competitive advantages have placed Business Intelligence (BI) software at the top of the technology priority list [2].

Data Warehousing Institute (DWI) that works in the field of education data warehouse and BI, define BI as follows: processes, technology and tools needed to transform data into information, information into knowledge and knowledge into plans that lead to a lucrative business action [3]. Vodapalli in [4] says "BI brings people and data together, offering a variety of ways to see the information that backs fact-based decision making". In other words, BI uses a set of tools to collect different information about the organization in a data warehouse and to analyze the collected data. Employees and managers utilize the result of this analysis to make better decisions and to help the growth and profitability of their organization. In fact, BI is an analysis tool in order to automate decision making about business conditions, sales, customer demand, product preferences, etc. [3]. The key tasks of a BI system include smart exploration, defragmentation, aggregation and multidimensional analysis of data obtained from different information resources [2]. BI systems include two types of instruments; the tools of database management systems are the first group that analyze the databases and the second group as competitive intelligence tools, they help organizations in decision making of the market [5].

After having reviewed the literature, we came to the conclusion that we define BI as a solution which should be considered in both managerial and technical approaches in order to assist experts and all levels of managers in decision making and taking processes.

Although BI can help to improve the performance of organizations, according to different literature reviews there are many companies that have failed to successfully implement BI projects [6]. Hawking and Sellitto in [6], indicate that 60 percent of the BI projects fail due to poor planning, poor management of projects, undelivered business requirements or ones delivered with low quality.

Lukić et al., proposed a new methodology for building multi-dimensional datawarehousing and business intelligence systems for utility companies [7]. Their approach can be used by any utility company that wishes to take advantage of the principles.

Since these projects by nature have a high failure rate, the identification of Critical Success Factors (CSF) of the projects can be important and can have a valuable role in the successful implementation of the system and in reducing the failure rate [1]. CSFs are a set of conditions, characteristics and variables that are defined in 
all fields and, if managed carefully, lead to organizational success. CSFs can help ensure the success of the BI implementation in an arbitrary organization.

In [2], Yeoh and Koronios said if BI shareholders reach an understanding of CSF, they can optimize their resources and efforts by focusing on critical factors that contribute to the successful implementation of the system. Farrokhi and Pokorádi [8] believed that these factors should be carefully considered by senior managers and BI project managers of companies that are evaluating the readiness of their organizations.

These factors will be much more effective, if used in accordance with their importance in various stages of implementation [8]. In this study, we used the factors presented in the paper of Farrokhi and Pokorádi [9] and listed in Figure 1. The authors in [9] have categorized the factors into both organizational and technical factors.

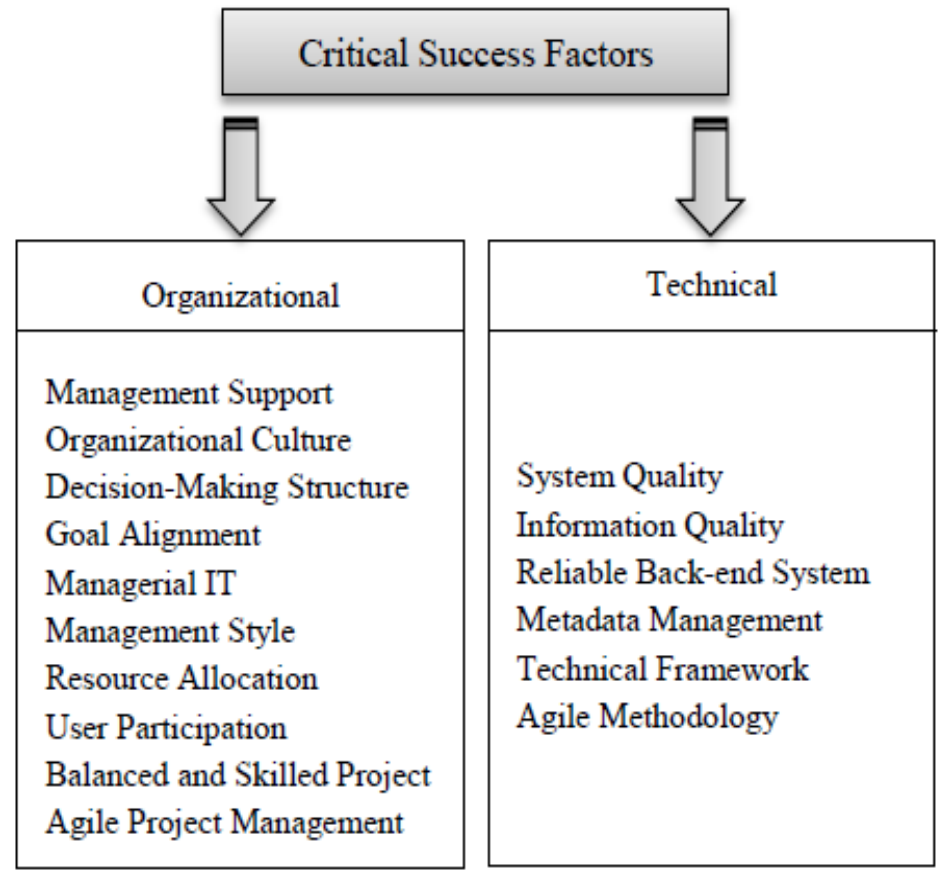

Figure 1

Critical Success Factors [8]

In [3] Farrokhi and Pokorádi reviewed the necessities for building a model to evaluate BI projects and they said that the BI readiness assessment had two main goals. Firstly, it shows the gap areas that are the reasons for the companyies not being ready to continue with the BI efforts. By identifying the BI readiness gaps, we will avoid wasting resources and time. Secondly, we can close these gaps by assessing and implementing BI with a high probability of success. 
In the [10] Hidayanto et. al. Used the Analytic Hierarchy Process (AHP) method and surveys of experts in the field of BI to estimate the weight criteria. They have used an assessment model of the Electronic Government Procurement (e-GP) to determine the level of each criterion. Then, by multiplying the weight of each criterion at their level, the readiness of the organization from the perspective of each criterion before the implementation of BI can be determined. In [11] factors affecting the readiness of BI were examined in three different organizations: IT, education and commercial. In the paper, the partial least squares method was used for analysis and to model the readiness of the organization. They examined the positive and negative effects of criteria in these three organizations before implementing BI.

Farrokhi et al. in [12] examined various assessment methods of organization readiness to implement business intelligence. They divided the existing methods into three categories: probabilistic method, Multi Criteria Decision Making (MCDM) methods (such as fuzzy AHP, fuzzy ANP, fuzzy AHF, AHP, ANP and AHF) and hybrid methods. Hybrid methods, combine probabilistic and MCDM methods to benefit the advantages of both methods.Then, they compared all these methods through AHP. The results demonstrated that the hybrid methods are the better optionsto build evaluation models.

Rajesh Attri et al. in [13] provided a survey on Interpretive Structural Modeling (ISM) and says it is a suitable method to identify relationships between specific items that define a problem and Rao in [14] indicated that Graph Theory and Matrix Approach (GTMA) is a good method for the modeling of systems, network analysis, functional representation, conceptual modeling, diagnosis, etc.

In [12], the authors presented that the hybrid approach is the most appropriate method to assess the readiness of an organization before implementing BI. Also, according to the results of [13] and [14], the combination of ISM and GTMA are considered in this study for assessing the organization's readiness.

Overall, the main contribution of this paper is the usage of the ISM method to depict the relationship between the involved key factors in a successful implementation of BI projects. Then, by using the Graph Theory and Matrix Approach (GTMA), obtain an indicator to evaluate the organization's readiness before implementing BI projects.

The rest of this paper is organized as follows: In Section 2 the mathematical background is studied. More precisely the concepts of ISM and GTMA are introduced in Sections 2.1 and 2.2 subsequently. Section 3 defines the research process and works done. Section 4 is the conclusion of our research. 


\section{Mathematical Background}

In this section the concepts of ISM and GTMA methods are presented. These concepts will be utilized in the research process and works done.

\subsection{ISM Method}

ISM is an appropriate technique to analyze the impact of an element on other elements. This methodology investigates respectively complex relationship directions between the elements of the system. In other words, the means by which, the system can overcome the complexity of the elements [15]. In this study, we need to determine the relationship between factors in a successful implementation of BI and convert them to a graph for further analysis. In this case, ISM comes to help. The implementation of ISM in accordance to [13] includes the following steps:

\subsubsection{Structural Self Interaction Matrix (SSIM)}

Primarily, utilizing the suggestions of experts from industry and academia, the relationships between components is defined. Four symbols are used to determine the relationship between two factors ( $i$ and $j$ ):

$V$ : means factor $\mathrm{i}$ leads to factor $\mathrm{j}$ (factor $\mathrm{i}$ will influence factor $\mathrm{j}$ ).

A: means factor $\mathrm{j}$ leads to factor $\mathrm{i}$ (factor $\mathrm{i}$ will be influenced by factor $\mathrm{j}$ ).

$\mathrm{X}$ : for both direction relations (factors $\mathrm{i}$ and $\mathrm{j}$ will influence each other).

O: for no relation between the factors.

\subsubsection{Reachability Matrix}

To get a reachability matrix, the symbols of the SSIM matrix must be converted to zero and one. The reachability matrix is calculated according to the following rules:

I. If the $(i, j)$ cell in the SSIM is $V$, then the $(i, j)$ cell in the reachability matrix becomes 1 and the $(\mathrm{j}, \mathrm{i})$ cell becomes 0 .

II. If the $(i, j)$ cell in the SSIM is A, then the $(i, j)$ cell in the matrix becomes 0 and the $(\mathrm{j}, \mathrm{i})$ cell becomes 1 .

III. If the (i,j) cell in the SSIM is X, then the $(\mathrm{i}, \mathrm{j})$ cell in the matrix becomes 1 and the $(\mathrm{j}, \mathrm{i})$ cell also becomes 1 .

IV. If the $(i, j)$ cell in the SSIM is $\mathrm{O}$, then the $(\mathrm{i}, \mathrm{j})$ cell in the matrix becomes 0 and the $(\mathrm{j}, \mathrm{i})$ cell also becomes 0 .

After obtaining the primary reachability matrix, the property of transmissibility must be checked. This means that if $(\mathrm{i}, \mathrm{j})=1$ and $(\mathrm{j}, \mathrm{k})=1$, then $(\mathrm{i}, \mathrm{k})=1$. 


\subsubsection{Level Partitions}

The Reachability set for a factor is a collection which includes the factor itself and the factors that can be reached through this factor and the Antecedent set is a collection that includes the factors which can be reached through them to this factor and the factor itself.

For level partitioning, first we determine the Reachability set and Antecedent set from the Reachability Matrix for any factor. After determining the Reachability set and Antecedent set, similar elements in both sets of factors are detected (named Similar set). In the first iteration, the factor with the same elements in the Reachability set and the Similar set is placed on the first level. Then, this factor is removed from consideration and the iteration is repeated to determine the factors of the second level. This process is continued until the levels of all the factors are defined.

\subsubsection{Diagraph}

According to the levels of each factor and the final Reachability Matrix, an initial model of ISM, with regard to the transmissibility is drawn. Then, the final version of ISM is computed by removing the transmissibility of nodes. This graph shows the relationships between different factors. High-level factors are placed on top of the graph and low-level factors at the bottom of the graph.Then the node contents are replaced with factors to obtain ISM model.

\subsection{Graph Theory and Matrix Approach}

A graph $G=[N, L, f]$ is a 3-tuple consisting of a set of nodes $N$, a set of links $L$, and a mapping function $f: L \rightarrow N \times N$ which maps links into pairs of nodes. Nodes directly connected by a link are called adjacent nodes [16]. When the node-pair order does not matter in linking the node pair, $G$ is an undirected graph. In an undirected graph, $p_{i} \sim p_{j}$ is equivalent to $p_{j} \sim p_{i}$, But in direct graph, a link defined by the node pair $\left(p_{i} ; p_{j}\right)$ is not the same as a link defined by node pair $\left(p_{j} ; p_{i}\right)$. In fact, both links may exist in a directed graph [16]. Adjacency matrix A shows the number of links directly connecting node $i$ to node $j$. This number is stored at row $i$, column $j$ of the matrix [16].

In this section, we transform the ISM diagraph to a matrix and by analyzing it; we will obtain an index to assess the readiness of the organization for the successful implementation of BI. The routine for applying GTMA to the matrix is as follows:

first, the Relative Importance Matrix (RIM), B from ISM digraph is defined. B is a binary matrix $\left(b_{i j}\right)$, where $b_{i j}$ represents the relative importance between factors $i$ and $j$ such that [14]: 
$\boldsymbol{B}=\left\{\begin{array}{lc}b_{i j}=1 & \begin{array}{c}\text { if the } i-\text { th factor is more important than the } \\ j-\text { th factor for a given machining operation. }\end{array} \\ & \text { otherwise }\end{array}\right.$

In other words, RIM is similar to the adjacency matrix in graph theory. In this matrix, all diagonal elements have a value of 0 and non-diagonal elements have a value of either 0 or 1 . So, in this matrix only the relative importance among the factors is considered whilst the measures of the factors are not considered. To incorporate this, another matrix, called Characteristic Matrix (CM), is defined:

$\mathbf{C}=[\mathbf{A I}-\mathbf{B}]$

Where $\mathbf{I}$ is an identity matrix, and $\mathbf{A}$ is a variable representing the measure of the factors. All diagonal elements of $\mathbf{C}$ are considered equal which means the measures of all factors are equal. But this is not true in the real world. In this formula, the relative importance of one factor to the second factor $\left(b_{i j}\right)$ can adopt values greater than zero or one [15]. To solve this problem, another matrix (D) called Variable Characteristic Matrix (VCM), is developed:

$\mathbf{D}=[\mathbf{E}-\mathbf{F}]$

In this equation, $\mathbf{E}$ is a diagonal matrix with diagonal elements $e_{i}$, which indicates the presence or size of factor $i$. If a factor is excellent, then it is assigned a maximum value. Also, for a very low significant factor, a minimum value is assigned [14]. $\mathbf{F}$ is a matrix of which the off-diagonal elements are represented as $f_{i, j}$, instead of 1 , wherever the $i$-th factor has more relative importance than the $j$-th factor.

Due to the positive and negative values in matrix $\mathbf{D}$, when calculating the matrix determinant, there may be a number of statements in the determinant formula of the calculation which become zero and so we lose some of the information. So, the Variable Permanent Function (VPF) is defined instead of the determinant. This function is derived from a new matrix called the Permanent Matrix [14]:

$\mathbf{H}=[\mathbf{E}+\mathbf{F}]$

The Permanent function (Leibniz's formula) is as follows [17]:

$\operatorname{Per}(H)=\sum_{\pi \in \prod_{n}} a_{1 \pi(1)} a_{2 \pi(2)} \ldots a_{n \pi(n)}(5)$

Where

$\prod_{n}=\{\pi \mid \pi$ is permutation of $\{1,2, \ldots, n\}\}$ 
Permanent function is a standard matrix function that is used in combinatorial mathematics [14].

The index to assess the readiness of an organization for the successful implementation of BI is achieved by using the Permanent matrix, from the following equation:

\section{$B I I A R^{1}=$ the numerical value of the Permanent matrix of factors.}

The time complexity of this formula is $\mathrm{O}\left(\mathrm{N}^{*} \mathrm{~N}\right.$ !) and it is not appropriate in cases where $\mathrm{N}$ is high. Therefore, we utilized the optimized algorithm from $\mathrm{H}$. J. Ryser (1963) which has higher execution speed $\left(\mathrm{O}\left(\mathrm{N}^{2} 2^{\mathrm{N}}\right)\right)$ [17]. This formula is as follows:

$\operatorname{per}(A)=\sum_{t=0}^{n-1}(-1)^{t} \sum_{x \in \tau_{n-t}} r_{1}(X) r_{2}(X) \ldots r_{n}(X)$

where

$\tau_{k}=\left\{X \epsilon R^{n \times k} \mid X\right.$ consists of columns of $\left.A\right\}$

and

$r_{i}(X)=$ sum of row $i$ of matrix $X$

We adopted this algorithm from [17] and wrote it in C\# and all the calculations were done.

\section{Works Done}

In this study, an index is achieved for evaluating the readiness of the organization to implement BI by combining ISM and GTMA methods and by taking into account the views of experts in the field of BI. Our research design is provided in Figure 2.

As it can be seen in Figure 2, we studied different literature and detected the critical factors that are involved in a successful implementation of BI. We used the technical and organizational factors for the successful implementation of BI that were presented by [8] and listed in Figure 1.

Two series of questionnaires were prepared according to these CSFs. The first questionnaire was related to the ISM and the second one is for GTMA. To check the reliability and validity of the questionnaires, we calculated Cronbach's alpha [18], which was 0.885 and also experts confirmed the accuracy of the questionnaires.

\footnotetext{
${ }^{1}$ Business Intelligence Index of Assess Readiness
} 
Detect the factors that are involved in the successful implementation of Business Intelligence

\section{Y}

A questionnaire was prepared taking into consideration the factors extracted in the previous step and it was filled by BI experts

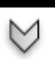

Extracting the ISM model based on the questionnaire of previous step and obtains the adjacency matrix of the ISM model

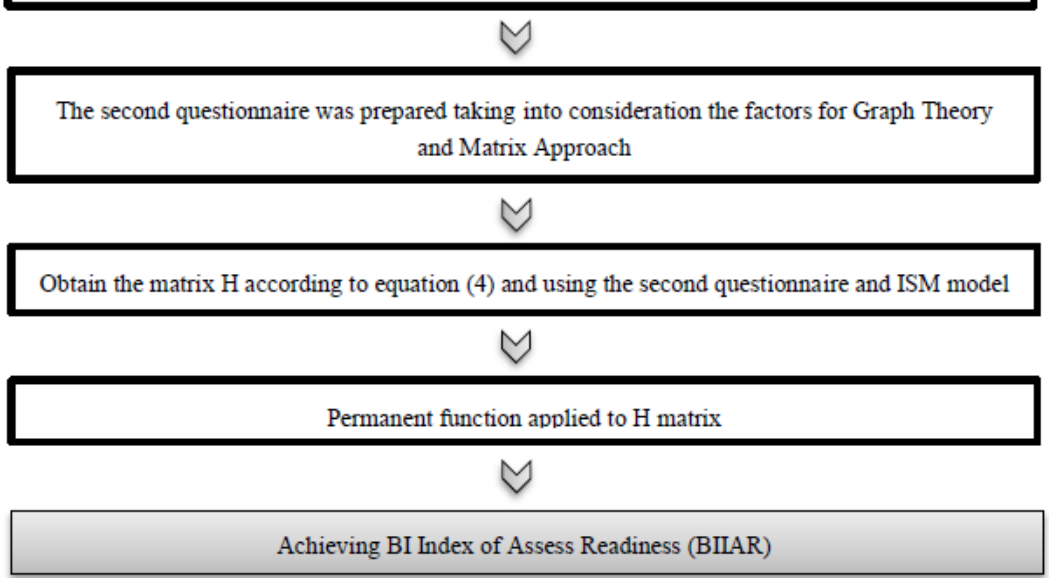

Figure 2

The block diagram of the proposed method

In the next phase questionnaires were completed by several specialists (five experts plus two academicians) in BI field. The relations between factors were extracted by applying ISM method and according to the experts' answers to the first questionnaire. In Table 2 the Final Reachability Matrix and in Table 3, Factor Levels resulted from ISM method are presented.

\section{Table 2}

Final Reachability Matrix for Sample Organization

\begin{tabular}{|c|c|c|c|c|c|c|c|c|c|c|c|c|c|c|c|c|}
\hline CF $\rightarrow$ & $\mathbf{1}$ & $\mathbf{2}$ & $\mathbf{3}$ & $\mathbf{4}$ & $\mathbf{5}$ & $\mathbf{6}$ & $\mathbf{7}$ & $\mathbf{8}$ & $\mathbf{9}$ & $\mathbf{1 0}$ & $\mathbf{1 1}$ & $\mathbf{1 2}$ & $\mathbf{1 3}$ & $\mathbf{1 4}$ & $\mathbf{1 5}$ & $\mathbf{1 6}$ \\
\hline CF1 & 1 & 1 & 1 & 1 & 1 & 1 & 1 & 1 & 1 & 1 & 1 & 1 & 1 & 1 & 1 & 1 \\
\hline CF2 & 1 & 1 & 1 & 1 & 1 & 1 & 1 & 1 & 1 & 1 & 1 & 1 & 1 & 1 & 1 & 1 \\
\hline CF3 & 1 & 1 & 1 & 1 & 0 & 1 & 1 & 1 & 1 & 1 & 1 & 1 & 1 & 1 & 1 & 1 \\
\hline CF4 & 1 & 1 & 1 & 1 & 0 & 1 & 1 & 1 & 1 & 1 & 1 & 1 & 1 & 1 & 1 & 1 \\
\hline CF5 & 1 & 1 & 1 & 1 & 1 & 1 & 1 & 1 & 1 & 1 & 1 & 1 & 1 & 1 & 1 & 1 \\
\hline CF6 & 1 & 1 & 1 & 1 & 1 & 1 & 1 & 1 & 1 & 1 & 1 & 1 & 1 & 1 & 1 & 1 \\
\hline CF7 & 1 & 1 & 1 & 1 & 0 & 1 & 1 & 1 & 1 & 1 & 1 & 1 & 1 & 1 & 1 & 1 \\
\hline
\end{tabular}




\begin{tabular}{|c|c|c|c|c|c|c|c|c|c|c|c|c|c|c|c|c|}
\hline CF8 & 1 & 1 & 1 & 1 & 0 & 0 & 0 & 1 & 1 & 1 & 1 & 1 & 1 & 1 & 1 & 1 \\
\hline CF9 & 1 & 1 & 1 & 1 & 0 & 1 & 1 & 1 & 1 & 1 & 1 & 1 & 1 & 1 & 1 & 1 \\
\hline CF10 & 1 & 1 & 1 & 1 & 1 & 1 & 1 & 1 & 1 & 1 & 1 & 1 & 1 & 1 & 1 & 1 \\
\hline CF11 & 1 & 1 & 1 & 1 & 0 & 1 & 0 & 1 & 0 & 1 & 1 & 1 & 1 & 1 & 1 & 1 \\
\hline CF12 & 1 & 1 & 1 & 1 & 0 & 1 & 1 & 1 & 0 & 0 & 1 & 1 & 1 & 1 & 1 & 1 \\
\hline CF13 & 1 & 1 & 1 & 1 & 0 & 1 & 1 & 1 & 0 & 0 & 1 & 1 & 1 & 1 & 1 & 1 \\
\hline CF14 & 1 & 0 & 1 & 1 & 0 & 0 & 1 & 1 & 0 & 0 & 1 & 1 & 1 & 1 & 1 & 1 \\
\hline CF15 & 1 & 1 & 1 & 1 & 0 & 1 & 1 & 1 & 0 & 1 & 1 & 1 & 1 & 1 & 1 & 1 \\
\hline CF16 & 1 & 1 & 1 & 1 & 0 & 1 & 1 & 1 & 1 & 1 & 1 & 1 & 1 & 1 & 1 & 1 \\
\hline
\end{tabular}

Table 3

Factor Levels

\begin{tabular}{|c|l|}
\hline Level & \multicolumn{1}{|c|}{ Factors } \\
\hline $\mathbf{1}$ & CF1,CF3,CF4,CF8,CF11,CF12,CF13,CF14,CF15,CF16 \\
\hline $\mathbf{2}$ & CF2,CF6,CF7, CF9, CF10 \\
\hline $\mathbf{3}$ & CF5 \\
\hline
\end{tabular}

According to these two tables, the Interpretive Structural Model is achieved. This model will be the input for GTMA. As the graph of ISM in our study was large and complex, we brought only the adjacency matrix of this model in Table 4 .

To apply GTMA, first we obtain the adjacency matrix of the ISM model. Then to create matrix $\mathbf{H}$ (equation 4), we form matrixes $\mathbf{E}$ and $\mathbf{F}$ by using the adjacency matrix of the ISM model and the results of the second questionnaire. $\mathbf{E}$ is a diagonal matrix with diagonal elements $e_{i}$, which indicates the importance of factors. The importance of factors was derived from the questionnaires completed by professionals. $\mathbf{F}$ is a matrix in which non-diagonal elements show the importance of one factor to another factor instead of 1 value in the adjacency matrix of ISM model. These values are derived from the second questionnaire too. Given the matrixes $\mathbf{E}$ and $\mathbf{F}$, the matrix $\mathbf{H}$ is obtained according to Equation 4.

Table4

Adjacency Matrix of ISM Model for Sample Organization

\begin{tabular}{|c|c|c|c|c|c|c|c|c|c|c|c|c|c|c|c|c|}
\hline $\mathbf{C F} \rightarrow$ & $\mathbf{1}$ & $\mathbf{2}$ & $\mathbf{3}$ & $\mathbf{4}$ & $\mathbf{5}$ & $\mathbf{6}$ & $\mathbf{7}$ & $\mathbf{8}$ & $\mathbf{9}$ & $\mathbf{1 0}$ & $\mathbf{1 1}$ & $\mathbf{1 2}$ & $\mathbf{1 3}$ & $\mathbf{1 4}$ & $\mathbf{1 5}$ & $\mathbf{1 6}$ \\
\hline $\mathbf{C F 1}$ & 9 & 4 & 0 & 5 & 0 & 4 & 4 & 4 & 2 & 3 & 3 & 2 & 0 & 2 & 3 & 0 \\
\hline CF2 & 4 & 8 & 3 & 2 & 0 & 3 & 0 & 4 & 3 & 2 & 0 & 0 & 0 & 0 & 0 & 0 \\
\hline CF3 & 3 & 0 & 7 & 2 & 0 & 0 & 3 & 2 & 3 & 4 & 2 & 3 & 0 & 0 & 3 & 3 \\
\hline CF4 & 4 & 3 & 4 & 8 & 0 & 0 & 0 & 3 & 0 & 2 & 0 & 0 & 0 & 0 & 0 & 0 \\
\hline CF5 & 4 & 0 & 0 & 0 & 7 & 3 & 3 & 0 & 3 & 2 & 3 & 4 & 3 & 3 & 0 & 2 \\
\hline CF6 & 0 & 2 & 3 & 2 & 3 & 7 & 2 & 3 & 3 & 4 & 3 & 3 & 0 & 0 & 0 & 3 \\
\hline CF7 & 0 & 3 & 0 & 4 & 0 & 0 & 9 & 3 & 0 & 3 & 4 & 4 & 4 & 3 & 2 & 0 \\
\hline CF8 & 0 & 0 & 0 & 2 & 0 & 0 & 0 & 8 & 3 & 0 & 5 & 4 & 0 & 3 & 0 & 0 \\
\hline CF9 & 4 & 4 & 5 & 0 & 0 & 0 & 4 & 5 & 8 & 3 & 4 & 3 & 3 & 3 & 4 & 4 \\
\hline
\end{tabular}




\begin{tabular}{|l|l|l|l|l|l|l|l|l|l|l|l|l|l|l|l|l|}
\hline CF10 & 0 & 4 & 4 & 3 & 0 & 5 & 4 & 4 & 5 & 6 & 3 & 3 & 3 & 2 & 3 & 4 \\
\hline CF11 & 0 & 3 & 0 & 0 & 0 & 0 & 0 & 3 & 0 & 0 & 7 & 4 & 4 & 0 & 5 & 4 \\
\hline CF12 & 4 & 5 & 4 & 3 & 0 & 0 & 0 & 0 & 0 & 0 & 4 & 8 & 0 & 0 & 2 & 0 \\
\hline CF13 & 4 & 0 & 3 & 2 & 0 & 0 & 0 & 2 & 0 & 0 & 3 & 2 & 7 & 3 & 4 & 0 \\
\hline CF14 & 3 & 0 & 4 & 0 & 0 & 0 & 0 & 0 & 0 & 0 & 3 & 5 & 4 & 6 & 0 & 0 \\
\hline CF15 & 4 & 0 & 3 & 0 & 0 & 0 & 0 & 0 & 0 & 0 & 2 & 0 & 5 & 3 & 8 & 4 \\
\hline CF16 & 4 & 3 & 4 & 0 & 0 & 0 & 3 & 4 & 0 & 4 & 3 & 2 & 2 & 0 & 3 & 6 \\
\hline
\end{tabular}

We brought matrix $\mathbf{H}$ for both sample organizations in Tables 5 and 6 .

Table5

Matrix $\mathrm{H}$ for First Organization

\begin{tabular}{|c|c|c|c|c|c|c|c|c|c|c|c|c|c|c|c|c|}
\hline CF $\rightarrow$ & $\mathbf{1}$ & $\mathbf{2}$ & $\mathbf{3}$ & $\mathbf{4}$ & $\mathbf{5}$ & $\mathbf{6}$ & $\mathbf{7}$ & $\mathbf{8}$ & $\mathbf{9}$ & $\mathbf{1 0}$ & $\mathbf{1 1}$ & $\mathbf{1 2}$ & $\mathbf{1 3}$ & $\mathbf{1 4}$ & $\mathbf{1 5}$ & $\mathbf{1 6}$ \\
\hline CF1 & 9 & 4 & 0 & 5 & 0 & 4 & 4 & 4 & 2 & 3 & 3 & 2 & 0 & 2 & 3 & 0 \\
\hline CF2 & 4 & 8 & 3 & 2 & 0 & 3 & 0 & 4 & 3 & 2 & 0 & 0 & 0 & 0 & 0 & 0 \\
\hline CF3 & 3 & 0 & 7 & 2 & 0 & 0 & 3 & 2 & 3 & 4 & 2 & 3 & 0 & 0 & 3 & 3 \\
\hline CF4 & 4 & 3 & 4 & 8 & 0 & 0 & 0 & 3 & 0 & 2 & 0 & 0 & 0 & 0 & 0 & 0 \\
\hline CF5 & 4 & 0 & 0 & 0 & 7 & 3 & 3 & 0 & 3 & 2 & 3 & 4 & 3 & 3 & 0 & 2 \\
\hline CF6 & 0 & 2 & 3 & 2 & 3 & 7 & 2 & 3 & 3 & 4 & 3 & 3 & 0 & 0 & 0 & 3 \\
\hline CF7 & 0 & 3 & 0 & 4 & 0 & 0 & 9 & 3 & 0 & 3 & 4 & 4 & 4 & 3 & 2 & 0 \\
\hline CF8 & 0 & 0 & 0 & 2 & 0 & 0 & 0 & 8 & 3 & 0 & 5 & 4 & 0 & 3 & 0 & 0 \\
\hline CF9 & 4 & 4 & 5 & 0 & 0 & 0 & 4 & 5 & 8 & 3 & 4 & 3 & 3 & 3 & 4 & 4 \\
\hline CF10 & 0 & 4 & 4 & 3 & 0 & 5 & 4 & 4 & 5 & 6 & 3 & 3 & 3 & 2 & 3 & 4 \\
\hline CF11 & 0 & 3 & 0 & 0 & 0 & 0 & 0 & 3 & 0 & 0 & 7 & 4 & 4 & 0 & 5 & 4 \\
\hline CF12 & 4 & 5 & 4 & 3 & 0 & 0 & 0 & 0 & 0 & 0 & 4 & 8 & 0 & 0 & 2 & 0 \\
\hline CF13 & 4 & 0 & 3 & 2 & 0 & 0 & 0 & 2 & 0 & 0 & 3 & 2 & 7 & 3 & 4 & 0 \\
\hline CF14 & 3 & 0 & 4 & 0 & 0 & 0 & 0 & 0 & 0 & 0 & 3 & 5 & 4 & 6 & 0 & 0 \\
\hline CF15 & 4 & 0 & 3 & 0 & 0 & 0 & 0 & 0 & 0 & 0 & 2 & 0 & 5 & 3 & 8 & 4 \\
\hline CF16 & 4 & 3 & 4 & 0 & 0 & 0 & 3 & 4 & 0 & 4 & 3 & 2 & 2 & 0 & 3 & 6 \\
\hline
\end{tabular}

Table 6

Matrix $\mathrm{H}$ for Second Organization

\begin{tabular}{|c|c|c|c|c|c|c|c|c|c|c|c|c|c|c|c|c|}
\hline CF $\rightarrow$ & $\mathbf{1}$ & $\mathbf{2}$ & $\mathbf{3}$ & $\mathbf{4}$ & $\mathbf{5}$ & $\mathbf{6}$ & $\mathbf{7}$ & $\mathbf{8}$ & $\mathbf{9}$ & $\mathbf{1 0}$ & $\mathbf{1 1}$ & $\mathbf{1 2}$ & $\mathbf{1 3}$ & $\mathbf{1 4}$ & $\mathbf{1 5}$ & $\mathbf{1 6}$ \\
\hline CF1 & 7 & 4 & 0 & 4 & 0 & 4 & 3 & 3 & 5 & 3 & 4 & 4 & 0 & 3 & 3 & 0 \\
\hline CF2 & 2 & 7 & 3 & 4 & 0 & 4 & 0 & 4 & 3 & 3 & 0 & 0 & 0 & 0 & 0 & 0 \\
\hline CF3 & 2 & 0 & 6 & 3 & 0 & 0 & 3 & 3 & 5 & 4 & 3 & 3 & 0 & 0 & 4 & 3 \\
\hline CF4 & 2 & 2 & 3 & 5 & 0 & 0 & 0 & 3 & 0 & 3 & 0 & 0 & 0 & 0 & 0 & 0 \\
\hline CF5 & 1 & 0 & 0 & 0 & 7 & 4 & 3 & 0 & 4 & 4 & 4 & 4 & 3 & 3 & 0 & 4 \\
\hline CF6 & 0 & 2 & 2 & 2 & 2 & 7 & 4 & 4 & 4 & 3 & 3 & 4 & 0 & 0 & 0 & 4 \\
\hline CF7 & 0 & 3 & 0 & 3 & 0 & 0 & 5 & 4 & 0 & 3 & 3 & 3 & 3 & 2 & 2 & 0 \\
\hline CF8 & 0 & 0 & 0 & 3 & 0 & 0 & 0 & 6 & 4 & 0 & 4 & 3 & 0 & 2 & 0 & 0 \\
\hline CF9 & 1 & 3 & 1 & 0 & 0 & 0 & 2 & 2 & 8 & 4 & 3 & 3 & 3 & 3 & 4 & 3 \\
\hline CF10 & 0 & 3 & 2 & 3 & 0 & 3 & 3 & 3 & 2 & 6 & 4 & 4 & 3 & 4 & 4 & 4 \\
\hline CF11 & 0 & 3 & 0 & 0 & 0 & 0 & 0 & 2 & 0 & 0 & 7 & 4 & 4 & 0 & 4 & 3 \\
\hline
\end{tabular}




\begin{tabular}{|l|l|l|l|l|l|l|l|l|l|l|l|l|l|l|l|l|}
\hline CF12 & 2 & 2 & 3 & 2 & 0 & 0 & 0 & 0 & 0 & 0 & 2 & 6 & 0 & 0 & 3 & 0 \\
\hline CF13 & 3 & 0 & 3 & 4 & 0 & 0 & 0 & 3 & 0 & 0 & 2 & 2 & 6 & 3 & 3 & 0 \\
\hline CF14 & 3 & 0 & 3 & 0 & 0 & 0 & 0 & 0 & 0 & 0 & 3 & 3 & 3 & 5 & 0 & 0 \\
\hline CF15 & 3 & 0 & 2 & 0 & 0 & 0 & 0 & 0 & 0 & 0 & 2 & 0 & 3 & 4 & 6 & 4 \\
\hline CF16 & 3 & 3 & 3 & 0 & 0 & 0 & 3 & 5 & 0 & 2 & 3 & 4 & 3 & 0 & 2 & 6 \\
\hline
\end{tabular}

Finally,permanent function applied to matrix $\mathbf{H}$ and BIIAR is obtained for both organizations. This index shows the readiness of each organization to implement BI.

\section{Conclusion}

Before the implementation of $\mathrm{BI}$, the readiness of the organization must be evaluated to minimize waste of costs and resources. In this study, we examined two utility companies with about 1,000 and 1,200 employees, in which their tasks are electricity transmission and distribution.

We applied the Interpretive Structural Modeling (ISM), Graph Theory and Matrix Approach (GTMA) to derive a measure to check the readiness of two sample organizations before implementing BI. The research design is demonstrated in Figure 2.

The adjacency matrix of the ISM model was illustrated in Table 4 and shows the relationship between the factors. GTMA applied to the ISM and the BI Index of Assess Readiness (BIIAR) for each organization was obtained to show the readiness of the organization before implementing BI. The result of GTMA which were applied to both organizations in this research is as follows:

$$
\begin{aligned}
& \text { BIIAR for first organization }=21.348 \times 10^{17} \\
& \text { BIIAR for second organization }=3.337 \times 10^{17}
\end{aligned}
$$

Beacuse of the BIIAR for the first organization is bigger, it will be relatively successful than the second organization in implementing BI project.

In an ideal situation, all values in matrix $\mathbf{H}$ will be maximum and so BIIAR will be $92.2337 \times 10^{17}$. But in the worst situation all values in matrix $\mathrm{H}$ will be minimum and BIIAR will be $6.3747 \times 10^{8}$. As you can see, the first organization is in better situation than second one, but still is far from the ideal.

The algorithm will produce different results according to society's culture and economy. Our suggestion is to implement the proposed algorithm in an organization that has already implemented BI successfully. Then the BIIAR rate obtained for that organization will be a standard for other organizations in the given society. Also, we can use an average from BIIARs of several successful organizations as a measure to obtain more accurate standards. 


\section{References}

[1] Zare Ravasan, A., \& Rabiee Savoji, s. (2014) Identification and Classification of Business Intelligence System Implementation Projects' Critical Success Factors in Iran. International Journal of Business Intelligence Research (5)3, 41-57

[2] Yeoh, W., \& Koronios, A. (2010) Critical Success Factors for business intelligence systems. Journal of Computer Information Systems, 50(3) 2332

[3] Farrokhi, v., \& Pokorádi, L. (2012) The Necessities for Building a Model to Evaluate Business Intelligence Projects- Literature Review. International Journal of Computer Science \& Engineering Survey, 3, No. 2

[4] Vodapalli, k. (2009) Critical Success Factors of BI Implementation (Master's Thesis) IT University of Copenhagen

[5] Babazadeh sangar, A., \& Noorminshah Binti Iahad, A. (2013) Critical Factors That Affect the Success of Business Intelligence Systems (BIS) Implementation in an Organization. International Journal of Scientific \& Technology Research (2)2

[6] Hawking, P., \& Sellitto, C. (2010). Business Intelligence (BI) Critical Success Factors. 21st Australasian Conference on Information Systems. Brisbane

[7] Lukić, J., Radenković, M., Despotović-Zrakić,M., Labus, A., Bogdanović, Z. (2016) A hybrid approach to building a multi-dimensional business intelligence system for electricity grid operators, Utilities Policy, Vol. 41, pp. $95-106$

[8] Farrokhi, v., \& Pokorádi, L. (2013) Organizational and Technical Factors for Implementing Business Intelligence. Fascicle of Management and Technological Engineering, ISSUE \#1

[9] Naderinejad, M., Tarokh, M. J., \& Poorebrahimi, A. (2014) Recognition and Ranking Critical Success Factors of Business Intelligence in Hospitals - Case Study: Hasheminejad Hospital. International Journal of Computer Science \& Information Technology, 6, No 2

[10] Achmad Nizar Hidayanto, \& Robertus Kristianto, \& Muhammad Rifki Shihab. (2012) Business Intelligence Implementation Readiness: A Framework Development and Its Application to Small Medium Enterprises (SMEs) International Research Symposium in Service Management (IRSSM-3) At Bejing, China

[11] Hejazi, A., \& Abdolvand, N., \& Harandi, S. R. (2016) Assessing The Organizational Readiness For Implementing BI Systems. International Journal of Information Technology Convergence and Services (IJITCS) Vol. 6, No. 1 
[12] Farrokhi, V., \& Pokorádi, L. (2012) a comparative analysis of evaluation methods for readiness of business intelligence project. Proceedings of the $13^{\text {th }}$ Conference on Mathematics and its Applications. University "Politehnica" of Timisoara, November, I3

[13] Rajesh Attri, Nikhil Dev, \& Vivek Sharma (2013) Interpretive Structural Modelling (ISM) Approach: An Overview. Research Journal of Management Sciences, 2(2) 3-8

[14] Rao, RV. (2007) Decision Making in the Manufacturing Environment: Using Graph Theory and Fuzzy Multiple Attribute Decision Making Methods (pp. 7-24) Springer, London

[15] Alam Tabriz, A., Talai, H., \& Moradi, E. (2013) Evaluating the Key Factors of Successful Implementation of World Class Manufacturing Using an Integrated Approach of Interpretive Structural Modeling(ISM), Graph Theory and Matrix Approach (GTMA): A Case Study for Iran Khodro and Saipa in Iran. Journal of Industrial Management (5)10, Number 1

[16] Pokorádi, L. (2013) Graph Theoretical Investigation of Network Structure System. 10th International Multidisciplinary Conference. 22-24

[17] RyserH. J. (1957) "Combinatorial properties of matrices of zeros and ones," Canadian Journal of Mathematics, Vol. 9, pp. 371-377

[18] Cronbach, L. J. (1951) "Coefficient Alpha and the Internal Structure of Tests," Psychometrika, Vol. 16, pp. 97-334 